\title{
The Possible Association of Female Pattern Hair Loss and Alteration in Serum Cholecalciferol Level
}

\section{Mohammed S Al-Abadie ${ }^{1}$, Hussain Tukmatchy ${ }^{2}$, Ari Ahmad ${ }^{3}$, Anandadeep Mandal ${ }^{4}$, Patrick A Ball ${ }^{5}$ and Hana Morrissey ${ }^{6 *}$}

${ }^{1}$ Consultant Dermatologist, Department of Dermatology, Royal Wolverhampton

Hospital Trust, Wolverhampton, United Kingdom

${ }^{2}$ General Practice Clinical Lead, Henley Primary Care Centre, United Kingdom

${ }^{3}$ Department of Dermatology, Royal Wolverhampton Hospital Trust, Wolverhampton, United Kingdom

${ }^{4}$ Assistant Professor, Statistician, Birmingham Business School, University of

Birmingham, United Kingdom

${ }^{5}$ Professor of Pharmacy, School of Pharmacy, University of Wolverhampton, United

Kingdom

${ }^{6}$ Reader in Clinical Pharmacy, School of Pharmacy, University of Wolverhampton, United Kingdom

*Corresponding Author: Hana Morrissey, Reader in Clinical Pharmacy, School of Pharmacy, Faculty of Science and Engineering, University of Wolverhampton, City Campus, United Kingdom.
Received: October 06, 2020

Published: November 28, 2020

(C) All rights are reserved by Hana Morrissey., et al.

\section{Abstract}

Background: Vitamin D has a central role in neuronal, reproductive, autoimmune, infectious, and heart diseases. Female Pattern Hair Loss (FPHL) is described as a non-scarring, progressive thinning of the hair resulting from a decreasing ratio of terminal hairs to thinner vellus hairs.

Aim: To explore the possibility of vitamin D deficiency contribution to hair loss in women in the local population in West Midlands England.

Method: Records of women from different age groups who were treated in the dermatology outpatient clinic for any skin conditions and had their vitamin D status assessed as part of their usual care. In this study we also examine the impact of vitamin D levels and age on hair loss.

Results: The studies reviewed medical records of 48 patients diagnosed with dermatological conditions. Low levels of vitamin D significantly affects the loss of hair $(\mathrm{p}=0.046)$. The odds ratio estimated is 3.545 . Our results show that the impact of low vitamin $\mathrm{D}$ level is significantly prevalent for the age groups 35 - 45 and moderately impacted age group 55 - 65 years.

Conclusion: Vitamin D has a significant role in hair-loss process, and possibly early correction may have a role in prevention and treatment in women aged 35 - 45 year of age. .

Keywords: Female Pattern Hair Loss; FPHL; Vitamin D; Pre-menopausal women; Post-Menopausal Women; Baldness 


\section{Introduction}

Vitamin D has an important role in skeletal health. It is known to have a central role in neuronal, reproductive, autoimmune, infectious, and heart diseases [1]. There are two sources of this vitamin; exogenous from dietary consumption and endogenous, produced after the skin has been exposed to solar ultraviolet radiation, especially UVB [2]. Vitamin D2 is derived from ergosterol after ultraviolet irradiation, while 7-dehydrocholesterol (Provitamin D3) converts into previtamin D3 after UVB exposure through the skin [3]. The next step is $\alpha$-hydroxylation which occurs in many organs including the kidney. Finally, the active form, calcitriol (1,25-dihydroxycholecalciferol) is produced in the liver [4].

Female Pattern Hair Loss (FPHL) is described as a non-scarring, progressive thinning of the hair resulting from a decreasing ratio of terminal hairs to thinner vellus hairs. The process is described as follicular miniaturisation [5]. The typical presentation is a diffuse loss of hair density, primarily over the vertex, but occipital areas may be involved [6]. It is the most frequent cause of hair loss and a major cause for referral to a specialist dermatology service in the UK. The pathophysiology of FPHL is unclear and appears to be multifactorial. Although the contributions of a combination of androgens and genetic susceptibility in male androgenic alopecia (AGA) are well accepted, the degree to which these factors contribute to FPHL is less clear [7]. The condition is common and in Caucasian females the incidence increases with age. Norwood reported a prevalence of $19 \%$ in a series of almost 1000 cases [8]. The prevalence increases post-menopause. In Norwood's series, $29 \%$ of patients over 70 year had the condition [8]. Other studies have reported up to $38 \%$ in older Caucasians [6] whilst the incidence appears lower (around 5 - 7\%) in Asian women [9,10].

AGA has a similar presentation to FPHL with miniaturised hair follicles; however, the role of androgens in females remain poorly understood [6]. Hamilton introduced the concept of androgen involvement in male pattern baldness noting that AGA never occurs in males who have never progressed through puberty, and that its progression stopped in men who were castrated [11]. He also noted that men receiving testosterone replacement therapy were prone to balding. AGA was subsequently described to be a consequence of the direct effects of dihydrotestosterone (DHT) on the dermal papilla of susceptible hair follicles [12]. DHT is a metabolite of testosterone, that binds to androgen receptors in hair follicles. This causes upregulation of the genes responsible for the transformation of terminal hair follicles to miniaturized hair follicles [13].

A similar process has been suggested in the development of FPHL. This is supported by the observation that women with hyperandrogenism can develop early-onset FPHL [14]. However, most patients with FPHL exhibit no other signs or symptoms of hyperandrogenism and have normal androgen levels, suggesting a different cause. The age-related increase in FPHL suggests oestrogen may be a factor [6].

Vitamin D status was identified as an important contributory factor in FPHL in the reviewed studies, however it remains unclear if this result is applicable to the local population, in West Midlands England.

\section{Aim of the Study}

To explore the possibility of vitamin D deficiency contribution to hair loss in women in the local population, in West Midlands England.

\section{Methods}

This study was categorised as surveillance audit. Records of women from different age groups who were treated in the dermatology outpatient clinic for any skin conditions and had their vitamin D status assessed as part of their usual care. Vitamin D status was assessed by testing serum 25(OH)D levels [15] and was classified as mild, moderate or severe (Table 1).

Clinic Codes were allocated as "diffuse hair loss", "hair loss", "hair thinning" and "normal hair state" to all records (Table 2).

Data was analysed using Microsoft ${ }^{\circledR}$ Excel $^{\circledR}$ and IBM SPSS ${ }^{\circledR}$ version 26.

\section{Results}

There were total of 48 patients' records, who have dermatology diagnosis, reviewed for vitamin D level test results. Out of those records there were $31 \%$ of all patients' records classed as having in- 


\begin{tabular}{|c|c|}
\hline Vitamin D status & $\begin{array}{c}\text { Serum 25-hydroxyvitamin D } \\
\text { level (nmol/L) }\end{array}$ \\
\hline Severe vitamin D deficiency & $<12$ \\
\hline Vitamin D deficiency & $12-25$ \\
\hline Vitamin D insufficiency & $25-50$ \\
\hline Marginal vitamin D status & $50-75$ \\
\hline Vitamin D sufficiency & $75-150$ \\
\hline Risk of toxicity & $>375$ \\
\hline
\end{tabular}

Table 1: Staging of vitamin D status used in this study [15].

\begin{tabular}{|c|c|}
\hline State & Definition \\
\hline Diffuse Hair Loss & Excessive FPHL hair loss \\
\hline Hair Loss & Moderate FPHL hair loss \\
\hline Hair Thinning & Mild FPHL hair loss \\
\hline Normal hair state & Normal hair no hair loss diagnosis \\
\hline
\end{tabular}

Table 2: Definitions of clinic code used in this study. sufficient or deficient vitamin D levels where $80 \%$ of those had hair loss (60\% and $20 \%$ respectively) and additional $36 \%$ with marginal vitamin D status where $60 \%$ of those had hair loss compared to $44 \%$ of all patients with sufficient vitamin D levels (Figure 1).

Figure 2 demonstrate that hair loss is more prevalent in females with suboptimal vitamin D (69\% of all patients) than females with normal vitamin D (44\%).

Using a binary logistic model, we examine the impact of vitamin D on hair loss. Table 3 shows that low levels of vitamin D significantly affects the loss of hair. The odds ratio estimated is 3.545 . This indicates that vitamin D is likely to play a significant role in hair-loss treatment.

In this study we also examine the impact of age on hair loss. Table 4 shows that the impact of low vitamin D level is significantly prevalent for the age groups 35 - 45 and 55 - 65 .

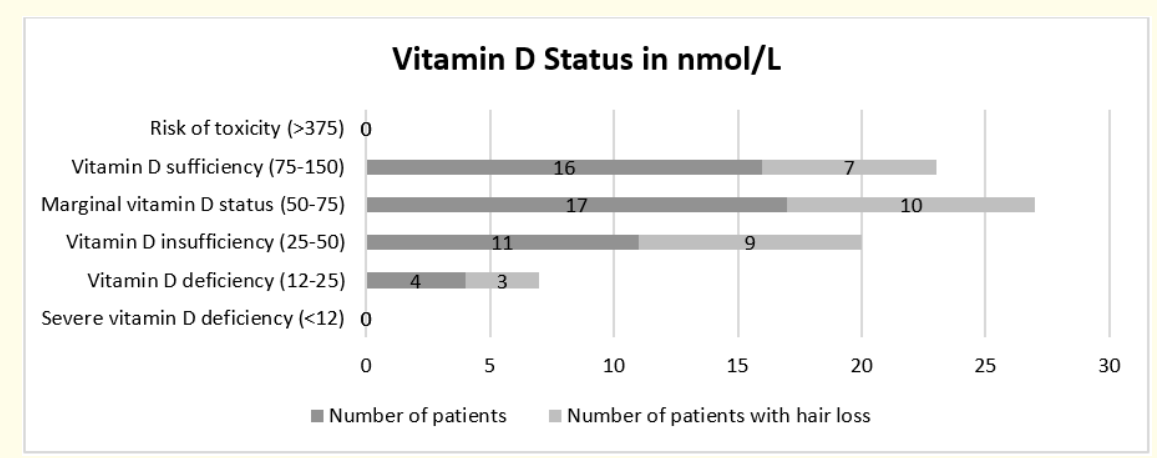

Figure 1: Vitamin D levels and co-occurrence of hair loss.

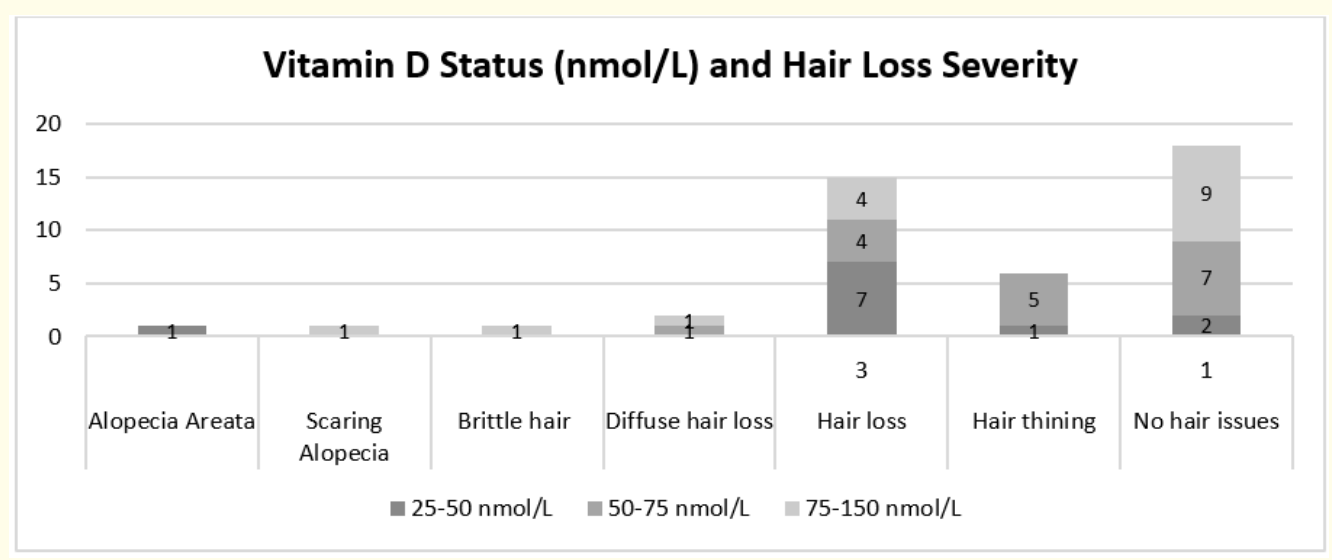

Figure 2: Comparing hair loss severity in women by vitamin D level status. 


\begin{tabular}{|c|c|c|c|}
\hline Factors & Odds Ratio & Std. error & p-value \\
\hline Constant & 0.846 & 0.410 & 0.683 \\
\hline Low Vitamin D Level & 3.545 & 0.625 & $0.043^{* *}$ \\
\hline Model Summary: & p-value & & \\
Chi-square Statistics & & & \\
\hline 4.347 & $0.037^{* *}$ & & \\
\hline
\end{tabular}

Table 3: Examining the impact of vitamin D on hair loss.

Note: ** represents significance at 5 percent level. The $\mathrm{p}$-value of the chi-square test suggests that Low Vitamin D level had a significant impact on the dependent variable, i.e. hair loss.

\begin{tabular}{|c|c|c|c|}
\hline Age Group & n & Chi-Square Statistic & p-value \\
\hline$\leq 25$ & 5 & 1.875 & 0.171 \\
\hline $26-35$ & 11 & 0.079 & 0.778 \\
\hline $36-45$ & 11 & 4 & $0.046^{* *}$ \\
\hline $46-55$ & 13 & 0.782 & 0.376 \\
\hline $56-65$ & 7 & 3 & $0.083^{*}$ \\
\hline$\geq 66$ & 1 & 1.333 & 0.248 \\
\hline
\end{tabular}

Table 4: Examining the significance of age.

Note: ${ }^{* *}$ represents significance at 5 percent level and

* represents significance at 10 percent level.

\section{Discussion}

Recent evidence has linked serum 25-hydroxycholecalciferol values with FPHL [16]. Monieb reported a low level of serum vitamin D in FPHL patients compared to controls [17]. In this study, $96.6 \%$ of FPHL patients were shown to have a low level of serum vitamin D; only $3.3 \%$ were measured within the normal range. A significant correlation was found between vitamin D level and the severity of FPHL. No link was found between the severity of hair loss and duration of the condition, or with the patient's age [17].

This was confirmed by Rasheed [18] and also Banihashemi [19], who also showed a correlation between severity of FPHL and lower concentrations of vitamin D, although these studies differed in their findings relating to the significance of a positive family history, which remains controversial [6]. Fawzi [20] identified lower numbers of vitamin D receptors in patients with both alopecia areata and AGA, but a higher concentration in patients with FPHL. The possible explanation could be due to the interaction between 17 beta oestradiol and 1,25 dihydroxycholecalciferol results in gene expression enhancement [21].

This study reviewed medical records of 48 patients diagnosed with dermatological conditions. In line with previous studies $[16,17,19,20]$ a low level of vitamin D was found to have a significant link with hair loss, suggesting a role in the causation and or pathogenesis of hair loss and thinning processes. That it has been identified in AA, AGA and FPHL suggests association rather than causation [20]. It is speculated that one factor may be emotional distress arising from altered appearance may prevent or reduce time spent outdoors [22] reducing exposure to UVB leading to reduced production of cholecalciferol.

However, a limitation is that none of these studies to date has accounted for seasonal variations in the level of dihydroxycholecalciferol [23]. Therefore, it would be an improvement in future studies to standardise the season during which the measurements of the measurement of the vitamin levels are made. This study sample, like most of the studies identified, is small and in a localised population. It is also becoming clear that in addition to vitamin D levels, the number of vitamin D receptors expressed on cells varies is altered in various skin conditions [24].

\section{Conclusion}

This study investigated the relationship between vitamin D levels and one type of hair loss, FPHL. The study showed a significant difference in hair loss between all age groups. This study showed vitamin D deficiency can be associated with hair loss in pre-menopausal women where the impact is highest for the age groups 35 - 45 followed by 55 - 65 years old. Whilst it clearly is a factor, there is more to be done to fully understand the mechanisms involved.

\section{Highlights}

1. Vitamin D deficiency is prevalent in local population, in West Midlands England. young females, 45 years of age and younger. 
2. Low vitamin D level correction may contribute to preventing or treating hair loss or thinning in young females, 45 years of age and younger.

\section{Bibliography}

1. Kechichian E and Ezzedine K. "Vitamin D and the skin: An update for dermatologists". American Journal of Clinical Dermatology 19.2 (2018): 223-235.

2. Chen TC., et al. "Factors that influence the cutaneous synthesis and dietary sources of vitamin D". Archives of Biochemistry and Biophysics 460.2 (2007): 213-217.

3. Tripkovic L., et al. "Comparison of vitamin D2 and vitamin D3 supplementation in raising serum 25-hydroxyvitamin D status: A systematic review and meta-analysis". The American Journal of Clinical Nutrition 95.6 (2012): 1357-1364.

4. Lucas RM., et al. "Vitamin D and immunity". F1000 Prime Reports 6 (2014): 118.

5. Messenger AG and Sinclair R. "Follicular miniaturization in female pattern hair loss: Clinicopathological correlations". British Journal of Dermatology 155.5 (2006): 926-930.

6. Vujovic A and Del Marmol V. "The female pattern hair loss: Review of etiopathogenesis and diagnosis". BioMed Research International (2014): 767628.

7. Yip L., et al. "Role of genetics and sex steroid hormones in male androgenetic alopecia and female pattern hair loss: An update of what we now know". Australasian Journal of Dermatology 52.2 (2011): 81-88.

8. Norwood OT. "Incidence of female androgenetic alopecia (female pattern alopecia)”. Dermatologic Surgery 27.1 (2001): 53-54.

9. Paik J., et al. "The prevalence and types of androgenetic alopecia in Korean men and women". British Journal of Dermatology 145.1 (2001): 95-99.

10. Wang TL., et al. "Prevalence of androgenetic alopecia in china: A community-based study in six cities". British Journal of Dermatology 162.4 (2010): 843-847.

11. Hamilton JB. "The relationship between common baldness and male sex hormones". Transactions of the New York Academy of Sciences (1946).
12. Whiting DA. "Possible mechanisms of miniaturization during androgenetic alopecia or pattern hair loss". Journal of the American Academy of Dermatology 45.3 (2001): 81.

13. Vexiau P., et al. "Role of androgens in female-pattern androgenetic alopecia, either alone or associated with other symptoms of hyperandrogenism". Archives of Dermatological Research 292.12 (2000): 598-604.

14. Price VH. "Androgenetic alopecia in women". Journal of Investigative Dermatology Symposium Proceedings 8.1 (2003): 24-27.

15. Lips P. "Vitamin D deficiency and secondary hyperparathyroidism in the elderly: Consequences for bone loss and fractures and therapeutic implications". Endocrine Reviews 22.4 (2001): 477-501.

16. El-Mongy N., et al. "Serum 25-hydroxy vitamin D3 level in Egyptian patients with alopecia areata". Journal of the Egyptian Women's Dermatologic Society 10.1 (2013).

17. Moneib H., et al. "Possible association of female-pattern hair loss with alteration in serum 25-hydroxyvitamin D levels". Egyptian Journal of Dermatology and Venerology 34.1 (2014): 15-20.

18. Rasheed H., et al. "Serum ferritin and vitamin D in female hair loss: Do they play a role?" SPP 26.2 (2013): 101-107.

19. Banihashemi M., et al. "Serum vitamin D3 level in patients with female pattern hair loss". International Journal of Trichology 8.3 (2016): 116-120.

20. Fawzi MMT., et al. "Assessment of vitamin D receptors in alopecia areata and androgenetic alopecia". Journal of Cosmetic Dermatology 15.4 (2016): 318-323.

21. Hayes CE., et al. "The immunological functions of the vitamin D endocrine system". Molecular and Cellular Biology 49.2 (2003): 277-300.

22. Cash TF., et al. "Psychological effects of androgenetic alopecia on women: Comparisons with balding men and with female control subjects". Journal of the American Academy of Dermatology 29.4 (1993): 568-575.

23. Maxwell JD. "Seasonal variation in vitamin D". Proceedings of the Nutrition Society 53.3 (1994): 533-543. 
The Possible Association of Female Pattern Hair Loss and Alteration in Serum Cholecalciferol Level

24. Demay MB. "The hair cycle and vitamin D receptor". Archives of Biochemistry and Biophysics 523.1 (2012): 19-21.

\section{Assets from publication with us}

- Prompt Acknowledgement after receiving the article

- Thorough Double blinded peer review

- Rapid Publication

- Issue of Publication Certificate

- High visibility of your Published work

Website: www.actascientific.com/

Submit Article: www.actascientific.com/submission.php Email us: editor@actascientific.com

Contact us: +919182824667 